PROCEEDINGS OF THE

AMERICAN MATHEMATICAL SOCIETY

Volume 137, Number 12, December 2009, Pages 4007-4017

S 0002-9939(09)09994-8

Article electronically published on July 30, 2009

\title{
JORDAN GRADINGS ON EXCEPTIONAL SIMPLE LIE ALGEBRAS
}

\author{
ALBERTO ELDUQUE
}

(Communicated by Gail R. Letzter)

\begin{abstract}
Models of all the gradings on the exceptional simple Lie algebras induced by Jordan subgroups of their groups of automorphisms are provided.
\end{abstract}

\section{INTRODUCTION}

Given a simple Lie algebra $\mathfrak{g}$ and a complex Lie group $G$ with $\operatorname{Int}(\mathfrak{g}) \leq G \leq$ $\operatorname{Aut}(\mathfrak{g})$ (here $\operatorname{Int}(\mathfrak{g})$ denotes the group of inner automorphisms and $\operatorname{Aut}(\mathfrak{g})$ the group of all the automorphisms of $\mathfrak{g}$ ), an abelian subgroup $A$ of $G$ is a Jordan subgroup if Ale74:

(i) its normalizer $N_{G}(A)$ is finite,

(ii) $A$ is a minimal normal subgroup of its normalizer, and

(iii) its normalizer is maximal among the normalizers of those abelian subgroups satisfying (i) and (ii).

The Jordan subgroups are shown in [Ale74 to be elementary (that is, isomorphic to $\mathbb{Z}_{p} \times \cdots \times \mathbb{Z}_{p}$ for some prime number $p$ ), and they induce gradings, called Jordan gradings, on the Lie algebra $\mathfrak{g}$.

The classification of Jordan subgroups is given in Ale74 in two tables. Table 1 deals with the classical Lie algebras. Detailed models of the corresponding Jordan gradings are given in OV91, Chapter 3, §3.12]. On the other hand, Table 2 in Ale74 gives the classification of the Jordan subgroups for the exceptional Lie algebras (see also OV91, Chapter 3, §3.13]). Table 1 below summarizes some properties of these Jordan subgroups and of the corresponding Jordan gradings. In all of them, the zero homogeneous subspace is trivial.

It turns out that the Jordan gradings on the exceptional simple complex Lie algebras, with the exception of a $\mathbb{Z}_{5}^{3}$-grading on $E_{8}$, look like the gradings recently obtained (see [Eld08, Remark 5.30]) from gradings on an octonion algebra and from gradings on a different type of (nonunital) composition algebra: the Okubo algebras.

Received by the editors October 15, 2008, and, in revised form, April 5, 2009.

2000 Mathematics Subject Classification. Primary 17B25.

Key words and phrases. Jordan grading, simple, exceptional, Lie algebra, orthogonal decomposition.

The author was supported by the Spanish Ministerio de Educación y Ciencia and FEDER (MTM 2007-67884-C04-02) and by the Diputación General de Aragón (Grupo de Investigación de Álgebra).

(C)2009 American Mathematical Society Reverts to public domain 28 years from publication 


\begin{tabular}{|c|c|c|}
\hline $\mathfrak{g}$ & $A$ & $\operatorname{dim} \mathfrak{g}_{\alpha}(\alpha \neq 0)$ \\
\hline$G_{2}$ & $\mathbb{Z}_{2}^{3}$ & 2 \\
\hline$F_{4}$ & $\mathbb{Z}_{3}^{3}$ & 2 \\
\hline$E_{8}$ & $\mathbb{Z}_{5}^{3}$ & 2 \\
\hline$D_{4}$ & $\mathbb{Z}_{2}^{3}$ & 4 \\
\hline$E_{8}$ & $\mathbb{Z}_{2}^{5}$ & 8 \\
\hline$E_{6}$ & $\mathbb{Z}_{3}^{3}$ & 3 \\
\hline
\end{tabular}

TABLE 1. The exceptional Jordan gradings

The first purpose of this paper is to check that those gradings induced by octonion and Okubo algebras are indeed Jordan gradings.

However, checking that a given subgroup of the automorphism group of a simple Lie algebra is a Jordan subgroup is not an easy task, so a different approach will be followed which consists of proving the next result, which is of independent interest:

Main Theorem. Let $\mathbb{F}$ be an algebraically closed ground field of characteristic 0 . Then, up to equivalence:

(i) There is a unique $\mathbb{Z}_{2}^{3}$-grading on the simple Lie algebra of type $G_{2}$ over $\mathbb{F}$ such that $\operatorname{dim} \mathfrak{g}_{\alpha}=2$ for any $0 \neq \alpha \in \mathbb{Z}_{2}^{3}$.

(ii) There is a unique $\mathbb{Z}_{3}^{3}$-grading on the simple Lie algebra of type $F_{4}$ over $\mathbb{F}$ such that $\operatorname{dim} \mathfrak{g}_{\alpha}=2$ for any $0 \neq \alpha \in \mathbb{Z}_{3}^{3}$.

(iii) There is a unique $\mathbb{Z}_{5}^{3}$-grading on the simple Lie algebra of type $E_{8}$ over $\mathbb{F}$ such that $\operatorname{dim} \mathfrak{g}_{\alpha}=2$ for any $0 \neq \alpha \in \mathbb{Z}_{5}^{3}$.

(iv) There is a unique $\mathbb{Z}_{2}^{3}$-grading on the simple Lie algebra of type $D_{4}$ over $\mathbb{F}$ such that $\operatorname{dim} \mathfrak{g}_{\alpha}=4$ for any $0 \neq \alpha \in \mathbb{Z}_{2}^{3}$.

(v) There is a unique $\mathbb{Z}_{2}^{5}$-grading on the simple Lie algebra of type $E_{8}$ over $\mathbb{F}$ such that $\operatorname{dim} \mathfrak{g}_{\alpha}=8$ for any $0 \neq \alpha \in \mathbb{Z}_{2}^{5}$.

(vi) There is a unique $\mathbb{Z}_{3}^{3}$-grading on the simple Lie algebra of type $E_{6}$ over $\mathbb{F}$ such that $\operatorname{dim} \mathfrak{g}_{\alpha}=3$ for any $0 \neq \alpha \in \mathbb{Z}_{3}^{3}$.

Recall that two gradings $\mathfrak{g}=\bigoplus_{g \in G} \mathfrak{g}_{g}$ and $\mathfrak{g}=\bigoplus_{\gamma \in \Gamma} \mathfrak{g}_{\gamma}$ are said to be equivalent if there is an automorphism $\varphi$ of $\mathfrak{g}$ such that for any $g \in G$ with $\mathfrak{g}_{g} \neq 0$, there is a $\gamma \in \Gamma$ with $\varphi\left(\mathfrak{g}_{g}\right)=\mathfrak{g}_{\gamma}$.

As mentioned above, in the recent paper Eld08, some natural gradings on either octonion algebras or Okubo algebras over fields of characteristic $\neq 2,3$ have been used to construct some nice gradings on the exceptional simple Lie algebras. Okubo algebras constitute a class of eight dimensional nonunital composition algebras. They are then endowed with a nondegenerate quadratic multiplicative form $n$ (so that $n(x * y)=n(x) n(y)$ for any $x, y$ ), and this form is such that the associated polar form $n(x, y)=n(x+y)-n(x)-n(y)$ is associative: $n(x * y, z)=n(x, y * z)$ for any $x, y, z$. These algebras were introduced by S. Okubo Oku78 and have some 
remarkable features (see for instance [KMRT98, Chapter 8]). Actually, over fields of characteristic $\neq 2,3$, Okubo algebras are precisely the forms of the so-called pseudo-octonion algebra, which is the algebra defined over the subspace of traceless $3 \times 3$ matrices over a field containing the cubic roots of 1 with multiplication and norm given by

$$
x * y=\omega x y-\omega^{2} y x-\frac{\omega-\omega^{2}}{3} \operatorname{trace}(x y) 1, \quad n(x)=-\frac{1}{2} \operatorname{trace}\left(x^{2}\right)
$$

for any $x, y$, where $\omega \neq 1=\omega^{3}$. It is an interesting fact that in this way a nonassociative composition algebra appears inside the associative algebra of $3 \times 3$ matrices.

More precisely, the following gradings on exceptional simple Lie algebras were obtained in Eld08:

(1) A $\mathbb{Z}_{2}^{3}$-grading on any octonion algebra $\mathbb{O}$ induces a $\mathbb{Z}_{2}^{3}$-grading on the simple Lie algebras $\mathfrak{g}$ of derivations of $\mathbb{O}$ (of type $G_{2}$ ) and also a $\mathbb{Z}_{2}^{3}$-grading on the orthogonal simple Lie algebra $\hat{\mathfrak{g}}$ of the skew-symmetric maps relative to the norm of $\mathbb{O}$ (of type $D_{4}$ ), with $\mathfrak{g}_{0}=0=\hat{\mathfrak{g}}_{0}$ and such that $\mathfrak{g}_{\alpha}$ (respectively $\hat{\mathfrak{g}}_{\alpha}$ ) is a Cartan subalgebra of $\mathfrak{g}$ (resp. $\hat{\mathfrak{g}}$ ) for any $0 \neq \alpha \in \mathbb{Z}_{2}^{3}$. (See Eld08, Subsection 5.2], and note that in many respects $D_{4}$ is exceptional.)

(2) A $\mathbb{Z}_{3}^{2}$-grading on any Okubo algebra $\mathcal{O}$ induces a $\mathbb{Z}_{3}^{3}$-grading on some attached simple Lie algebras $\mathfrak{g}$ and $\hat{\mathfrak{g}}$ of types $F_{4}$ and $E_{6}$, with $\mathfrak{g}_{0}=0=\hat{\mathfrak{g}}_{0}$ and such that $\mathfrak{g}_{\alpha}$ (respectively $\hat{\mathfrak{g}}_{\alpha}$ ) is a two dimensional subalgebra of $\mathfrak{g}$ (respectively a three dimensional subalgebra of $\hat{\mathfrak{g}}$ ) with $\mathfrak{g}_{\alpha} \oplus \mathfrak{g}_{-\alpha}$ (respectively $\hat{\mathfrak{g}}_{\alpha} \oplus \hat{\mathfrak{g}}_{-\alpha}$ ) being a Cartan subalgebra of $\mathfrak{g}$ (resp. $\left.\hat{\mathfrak{g}}\right)$ for any $0 \neq \alpha \in \mathbb{Z}_{3}^{3}$. (See [Eld08, Subsection 5.3].)

(3) $\mathrm{A} \mathbb{Z}_{2}^{3}$-grading on each of two octonion algebras induces a $\mathbb{Z}_{2}^{5}$-grading on some attached simple Lie algebra $\mathfrak{g}$ of type $E_{8}$, with $\mathfrak{g}_{0}=0$ and such that $\mathfrak{g}_{\alpha}$ is a Cartan subalgebra of $\mathfrak{g}$ for any $0 \neq \alpha \in \mathbb{Z}_{2}^{5}$. (See [Eld08, Subsection 5.4].)

An immediate corollary of the Main Theorem is that indeed the gradings above obtained from gradings on octonion or Okubo algebras are Jordan gradings.

Actually, parts (i), (iv) and (v) of the Main Theorem follow from results by Hesselink. In fact, by [Hes82, Proposition 3.6], any grading of a simple Lie algebra $\mathfrak{g}$ over $\mathbb{F}$ with the properties of the gradings in the Main Theorem satisfies the property that for any $0 \neq \alpha$ in the grading group (which is $\mathbb{Z}_{p}^{r}$ for $p=2,3$ or 5 and $r=3$ or 5 ) the subspace

$$
\mathfrak{g}_{[\alpha]}=\bigoplus_{i=0}^{p-1} \mathfrak{g}_{i \alpha}
$$

is always a Cartan subalgebra of $\mathfrak{g}$. Now, by Hes82, Theorem 6.2], these gradings are unique (up to equivalence) in cases (i), (iv) and (v) of the Main Theorem, where $\mathfrak{g}_{\alpha}$ is a Cartan subalgebra for any $\alpha \neq 0$. For $E_{8}$ this was proved earlier in [Tho76], where it was shown that there is a unique (up to conjugation by automorphisms) Dempwolff decomposition of $E_{8}$.

Moreover, the gradings in parts (i), (iv) and (v) are all obtained from the natural $\mathbb{Z}_{2}^{3}$-grading on the algebra of octonions $\mathbb{O}$ (see [Eld98] and [Eld08]). The $\mathbb{Z}_{2}^{3}$-gradings on $G_{2}=\mathfrak{d e r} \mathbb{O}$ and on $D_{4}=\mathfrak{s o}(\mathbb{O})$ are just the gradings induced from the one in $\mathbb{O}$, while the $\mathbb{Z}_{2}^{5}$-grading on $E_{8}$ is obtained from the model of $E_{8}$ as a direct sum of two copies of the triality Lie algebra of the octonions (which is isomorphic to 
$\mathfrak{s o}(\mathbb{O}))$ and three copies of the tensor product of two copies of the octonions:

$$
E_{8}=(\mathfrak{t r i}(\mathbb{O}) \oplus \mathfrak{t r i}(\mathbb{O})) \oplus \iota_{0}(\mathbb{O} \otimes \mathbb{O}) \oplus \iota_{1}(\mathbb{O} \otimes \mathbb{O}) \oplus \iota_{2}(\mathbb{O} \otimes \mathbb{O})
$$

(see [Eld08] and the references therein). This model of $E_{8}$ is naturally $\mathbb{Z}_{2}^{2}$-graded, with the zero homogeneous part given by the direct sum of the two copies of the triality Lie algebra and the nonzero homogeneous parts given by the three copies of the tensor product of two copies of $\mathbb{O}$. And this $\mathbb{Z}_{2}^{2}$-grading is now refined by means of the $\mathbb{Z}_{2}^{3}$-grading of $\mathbb{O}$ to get a $\mathbb{Z}_{2}^{5}$-grading of $E_{8}$ with the required properties (see Eld08, §5.4] for the details).

Therefore, the rest of this paper will be devoted to proving parts (ii), (iii), and (vi) of the Main Theorem. In the process, very concrete models of the corresponding Jordan gradings will emerge.

As a consequence, detailed models of all the Jordan gradings in Table 2 of Ale74 (the exceptional Jordan gradings) are obtained.

To finish this introduction, note that all these gradings are related to the so-called orthogonal decompositions introduced in KKU81 (see KP94 and the references therein). For any of these gradings, if we denote by $\mathbb{P}\left(\mathbb{Z}_{p}^{r}\right)$ the projective space of dimension $r-1$ over the finite field $\mathbb{Z}_{p}$ and if for $0 \neq \alpha \in \mathbb{Z}_{p}^{r},[\alpha]$ denotes the corresponding point in $\mathbb{P}\left(\mathbb{Z}_{p}^{r}\right)$, then the subalgebras $\mathfrak{g}_{[\alpha]}$ in (1.1) are Cartan subalgebras of $\mathfrak{g}$, and the decomposition

$$
\mathfrak{g}=\bigoplus_{[\alpha] \in \mathbb{P}\left(\mathbb{Z}_{p}^{r}\right)} \mathfrak{g}_{[\alpha]}
$$

is a decomposition of $\mathfrak{g}$ into a direct sum of Cartan subalgebras which are orthogonal relative to the Killing form (as the homogeneous subspaces $\mathfrak{g}_{\alpha}$ and $\mathfrak{g}_{\beta}$ are always orthogonal unless $\beta=-\alpha$ ). That is, the decomposition in (1.2) is an orthogonal decomposition of $\mathfrak{g}$.

The next section will be devoted to proving the Main Theorem for the $\mathbb{Z}_{5}^{3}$ gradings on $E_{8}$ (part (iii)), and then Section 3 will deal with parts (vi) and (ii).

\section{2. $\mathbb{Z}_{5}^{3}$-GRADING ON $E_{8}$}

The purpose of this section is to prove part (iii) of the Main Theorem, that is:

Theorem 2.1. Let $\mathbb{F}$ be an algebraically closed field of characteristic 0 and let $\mathfrak{g}$ be the simple Lie algebra of type $E_{8}$ over $\mathbb{F}$. Then up to equivalence there is a unique $\mathbb{Z}_{5}^{3}$-grading of $\mathfrak{g}$ such that $\operatorname{dim} \mathfrak{g}_{\alpha}=2$ for any $0 \neq \alpha \in \mathbb{Z}_{5}^{3}$.

Proof. First note that by a dimension count $\mathfrak{g}_{0}=0$ holds. The proof will follow several steps.

Step 1. The construction of a suitable model of the simple Lie algebra of type $E_{8}$.

Let $V_{1}$ and $V_{2}$ be two vector spaces over $\mathbb{F}$ of dimension 5 , and consider the $\mathbb{Z}_{5}$-graded vector space

$$
\mathfrak{g}=\bigoplus_{i=0}^{4} \mathfrak{g}_{\bar{i}}
$$


where

$$
\begin{aligned}
\mathfrak{g}_{\overline{0}} & =\mathfrak{s l}\left(V_{1}\right) \oplus \mathfrak{s l}\left(V_{2}\right), \\
\mathfrak{g}_{\overline{1}} & =V_{1} \otimes \bigwedge^{2} V_{2}, \\
\mathfrak{g}_{\overline{2}} & =\bigwedge^{2} V_{1} \otimes \bigwedge^{4} V_{2}, \\
\mathfrak{g}_{\overline{3}} & =\bigwedge^{3} V_{1} \otimes V_{2}, \\
\mathfrak{g}_{\overline{4}} & =\bigwedge^{4} V_{1} \otimes \bigwedge^{3} V_{2} .
\end{aligned}
$$

(All the tensor products are considered over the ground field $\mathbb{F}$.) This is a $\mathbb{Z}_{5-}$ graded Lie algebra, with the natural action of the semisimple algebra $\mathfrak{g}_{\overline{0}}$ on each of the other homogeneous components and where the brackets between elements in different components are given by suitable scalar multiples of the only $\mathfrak{g}_{0}$-invariant possibilities. In this way, $\mathfrak{g}$ is the exceptional simple Lie algebra of type $E_{8}$. The details of the Lie multiplication have been computed in Dra05. This decomposition has received some attention lately Kos08.

Step 2. Up to conjugation in Aut $\mathfrak{g}$, there is a unique order 5 automorphism of the simple Lie algebra $\mathfrak{g}$ of type $E_{8}$ such that the dimension of the subalgebra of fixed elements is 48 .

Actually, as shown in [Kac90, §8.6], up to conjugation, the finite order automorphisms of $E_{8}$ are in one-to-one correspondence with subsets of nodes of the affine Dynkin diagram $E_{8}^{(1)}$ :

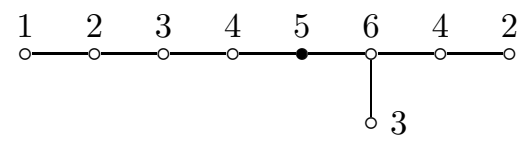

such that the sum of the integers that label the nodes in the subset is exactly 5 . Given such a subset of, say, $r$ nodes, the fixed subalgebra is the direct sum of the semisimple Lie algebra whose Dynkin diagram is obtained by removing from (2.4) the nodes in the subset and a center of dimension $r-1$. Now it is easy to see that the only possibility is the automorphism $\sigma$ obtained when considering the subset that consists exactly of the node with label 5 . In this case, one gets a $\mathbb{Z}_{5}$-grading of $\mathfrak{g}$ where $\mathfrak{g}_{0}$ is a direct sum of two copies of the simple Lie algebra of type $A_{4}$. The uniqueness shows us that, up to conjugation, $\sigma$ is the automorphism of $\mathfrak{g}$ such that its restriction to $\mathfrak{g}_{\bar{\imath}}$ (with notation as in Step 1) is $\xi^{i}$ times the identity, where $\xi$ is a fixed primitive fifth root of unity.

Step 3. Assume that $\mathfrak{g}=\bigoplus_{0 \neq \alpha \in \mathbb{Z}_{5}^{3}} \mathfrak{g}_{\alpha}$ is a $\mathbb{Z}_{5}^{3}$-graded simple Lie algebra of type $E_{8}$ with $\operatorname{dim} \mathfrak{g}_{\alpha}=2$ for any $0 \neq \alpha \in \mathbb{Z}_{5}^{3}$. The homogeneous spaces are given by the common eigenspaces of three commuting order 5 automorphisms $\sigma_{1}, \sigma_{2}$, and $\sigma_{3}$ of $\mathfrak{g}$ which generate a subgroup of Aut $\mathfrak{g}$ isomorphic to $\mathbb{Z}_{5}^{3}$.

3.1 $\left(\sigma_{1}\right)$ : Step 2 shows us that, without loss of generality, we may assume that $\sigma_{1}$ is the automorphism such that $\sigma_{1}(x)=\xi^{i} x$ for any $x \in \mathfrak{g}_{\bar{\imath}}$ (notation as in Step 1). $3.2\left(\sigma_{2}\right)$ : Consider now the order 5 automorphism $\sigma_{2}$. As it commutes with $\sigma_{1}$, the restriction $\left.\sigma_{2}\right|_{\mathfrak{g}_{\overline{0}}}$ is an automorphism of $\mathfrak{g}_{\overline{0}}$. Its order is then either 1 or 5 . Given a subset of automorphisms of $\mathfrak{g}$, let us denote by $\operatorname{Fix}(S)$ the subset of elements that 
are fixed by all the elements in $S$. Note that

$$
\operatorname{Fix}\left(\left.\sigma_{2}\right|_{\mathfrak{g}_{\overline{0}}}\right)=\operatorname{Fix}\left(\sigma_{1}, \sigma_{2}\right)=\bigoplus_{i=0}^{4} \mathfrak{g}_{i \alpha}
$$

for some $0 \neq \alpha \in \mathbb{Z}_{5}^{3}$ and that this subspace has dimension 8 . We conclude that $\left.\sigma_{2}\right|_{\mathfrak{g}_{\overline{0}}}$ has order 5. Since $\mathfrak{s l}\left(V_{1}\right)$ and $\mathfrak{s l}\left(V_{2}\right)$ are the only ideals of $\mathfrak{g}_{\overline{0}}$ and $\sigma_{2}$ induces a permutation of these two ideals of order 1 or 5 , it follows that both $\mathfrak{s l}\left(V_{1}\right)$ and $\mathfrak{s l}\left(V_{2}\right)$ are invariant under the action of $\sigma_{2}$; and since $\operatorname{dim} \operatorname{Fix}\left(\sigma_{1}, \sigma_{2}\right)$ is 8 , it turns out that the restriction of $\sigma_{2}$ to $\mathfrak{s l}\left(V_{i}\right)$ has order $5(i=1,2)$.

Recall (see [Jac62, Chapter IX]) that $\operatorname{Int}\left(\mathfrak{s l}\left(V_{i}\right)\right.$ is the group generated by the set $\left\{\exp \operatorname{ad}_{a}: a \in \mathfrak{s l}\left(V_{i}\right), a\right.$ nilpotent $\}$ and that the quotient $\operatorname{Aut}\left(\mathfrak{s l}\left(V_{i}\right)\right) / \operatorname{Int}\left(\mathfrak{s l}\left(V_{i}\right)\right)$ is a cyclic group of order 2. Since the order of the restriction $\left.\sigma_{2}\right|_{\mathfrak{s} l}\left(V_{i}\right)$ is 5 , this restriction belongs to $\operatorname{Int}\left(\mathfrak{s l}\left(V_{i}\right)\right), i=1,2$.

Therefore, there are nilpotent endomorphisms $a_{i j} \in \mathfrak{s l}\left(V_{i}\right), j=1, \ldots, m_{i}, i=$ 1,2 , such that

$$
\left.\sigma_{2}\right|_{\mathfrak{s l}\left(V_{i}\right)}=\exp \operatorname{ad}_{a_{i 1}} \cdots \exp \operatorname{ad}_{a_{i m_{i}}} .
$$

Hence, the restriction $\left.\sigma_{2}\right|_{\mathfrak{g}_{\overline{0}}}$ extends to the automorphism $\hat{\sigma}_{2}$ of $\mathfrak{g}$ given by the formula

$$
\hat{\sigma}_{2}=\exp \operatorname{ad}_{a_{11}} \cdots \exp \operatorname{ad}_{a_{1 m_{1}}} \exp \operatorname{ad}_{a_{21}} \cdots \exp \operatorname{ad}_{a_{2 m_{2}}} .
$$

Note that $\hat{\sigma}_{2}$ leaves invariant the subspaces $\mathfrak{g}_{\bar{i}}$, for $0 \leq i \leq 4$. Thus the automorphism $\hat{\sigma}_{2}^{-1} \sigma_{2}$ leaves invariant all the subspaces $\mathfrak{g}_{\bar{\imath}}$ and its restriction to $\mathfrak{g}_{0}$ is the identity. But each $\mathfrak{g}_{\overline{1}}$ is an irreducible module for $\mathfrak{g}_{\overline{0}}$, so Schur's Lemma shows that there is a nonzero scalar $\lambda \in \mathbb{F}$ such that

$$
\left.\hat{\sigma}_{2}^{-1} \sigma_{2}\right|_{\mathfrak{g}_{\overline{1}}}=\lambda 1 ;
$$

and, as $\mathfrak{g}_{\overline{1}}$ generates $\mathfrak{g}$ as a Lie algebra, it follows that the restriction of $\hat{\sigma}_{2}^{-1} \sigma_{2}$ to $\mathfrak{g}_{\bar{\imath}}$ is $\lambda^{i}$ times the identity map, where $\lambda^{5}=1$. Also note that given any endomorphism $a \in \mathfrak{s l}\left(V_{i}\right), \exp \operatorname{ad}_{a}=\operatorname{Ad}_{\exp a}$ on $\mathfrak{s l}\left(V_{i}\right)\left(\operatorname{Ad}_{g}(x)=g x g^{-1}\right.$ for any $g \in G L\left(V_{i}\right)$ and $\left.a \in \mathfrak{s l}\left(V_{i}\right)\right)$, while $\operatorname{ad}_{a}$ acts on each $\bigwedge^{j} V_{i}$ in the natural way, so that exp $\operatorname{ad}_{a}$ acts on $\wedge^{j} V_{i}$ as $\wedge^{j} \exp a\left(\right.$ where $\left.\left(\wedge^{j} f\right)\left(w_{1} \wedge \cdots \wedge w_{j}\right)=f\left(w_{1}\right) \wedge \cdots \wedge f\left(w_{j}\right)\right)$.

Consider the elements $b_{i j}=\exp a_{i j} \in S L\left(V_{i}\right)$, and let $b_{i}=b_{i 1} \cdots b_{i m_{i}}$. Then the restrictions of $\hat{\sigma}_{2}$ to $\mathfrak{s l}\left(V_{i}\right)(i=1,2)$ and $\mathfrak{g}_{\overline{1}}=V_{1} \otimes \bigwedge^{2} V_{2}$ are, respectively, the automorphism $\operatorname{Ad}_{b_{i}}$ and the linear isomorphism $b_{1} \otimes \wedge^{2} b_{2}$. If $b_{1}$ is changed to $\lambda b_{1}$, then we get a new automorphism $\tilde{\sigma}_{2}$ such that $\left.\tilde{\sigma}_{2}\right|_{\mathfrak{g}_{\overline{0}}}=\left.\hat{\sigma}_{2}\right|_{\mathfrak{g}_{\overline{0}}}=\left.\sigma_{2}\right|_{\mathfrak{g}_{\overline{0}}}$ and $\left.\tilde{\sigma}_{2}\right|_{\mathfrak{g}_{\mathfrak{1}}}=\left.\lambda \hat{\sigma}_{2}\right|_{\mathfrak{g}_{\mathfrak{1}}}=\left.\sigma_{2}\right|_{\mathfrak{g}_{\mathfrak{1}}}$. It follows that $\tilde{\sigma}_{2}=\sigma_{2}$ (recall that $\mathfrak{g}_{\overline{1}}$ generates $\left.\mathfrak{g}\right)$.

Summarizing the previous arguments, it has been proven that there are elements $b_{i} \in S L\left(V_{i}\right), i=1,2$, such that

$$
\left.\sigma_{2}\right|_{\mathfrak{s} l\left(V_{i}\right)}=\operatorname{Ad}_{b_{i}} \quad(i=1,2),\left.\quad \sigma_{2}\right|_{\mathfrak{g}_{\overline{1}}}=b_{1} \otimes \wedge^{2} b_{2} .
$$

Moreover, the order of $\sigma_{2}$ is 5 , so $\left(\left.\sigma_{2}\right|_{\mathfrak{g}_{0}}\right)^{5}=1$, which implies that $b_{i}^{5}=\lambda_{i} 1_{V_{i}}$ for some $0 \neq \lambda_{i} \in \mathbb{F}, i=1,2$. But also $\left(\left.\sigma_{2}\right|_{\mathfrak{g}_{\overline{1}}}\right)^{5}=1$, whence $\lambda_{1} \lambda_{2}^{2}=1$. Since $\mathbb{F}$ is algebraically closed, we can take scalars $\mu_{1}, \mu_{2} \in \mathbb{F}$ such that $\mu_{1}^{5}=\lambda_{1}^{-1}, \mu_{2}^{5}=\lambda_{2}^{-1}$ and $\mu_{1} \mu_{2}^{2}=1$. We may replace $b_{i}$ by $\mu_{i} b_{i}, i=1,2$, in (2.5) and hence assume that $b_{i}^{5}=1_{V_{i}}, i=1,2$.

Besides, for $i=1,2$, since $b_{i}^{5}=1, b_{i}$ is a diagonalizable endomorphism of $V_{i}$ whose eigenvalues are fifth roots of unity. Note that the subspace $\left\{x \in \mathfrak{s l}\left(V_{i}\right): b_{i} x b_{i}^{-1}=x\right\}$ has dimension at least 4 , because the endomorphisms which act diagonally on a basis 
of eigenvectors of $b_{i}$ commute with $b_{i}$. But if an eigenvalue of $b_{i}$ has multiplicity $\geq 2$, then the dimension above is strictly greater than 4 , and this contradicts the dimension of $\operatorname{Fix}\left(\sigma_{1}, \sigma_{2}\right)=\operatorname{Fix}\left(\left.\sigma_{2}\right|_{\mathfrak{g}_{0}}\right)$ being exactly 8 . Therefore, all the eigenvalues of $b_{i}$ have multiplicity 1 , and therefore a basis $\left\{v_{i 1}, \ldots, v_{i 5}\right\}$ of $V_{i}$ can be taken with $b_{i}\left(v_{i j}\right)=\xi^{j} v_{i j}(i=1,2, j=1,2,3,4,5)$. That is, the matrix of $b_{i}$ in this basis is precisely

$$
\left(\begin{array}{ccccc}
1 & 0 & 0 & 0 & 0 \\
0 & \xi & 0 & 0 & 0 \\
0 & 0 & \xi^{2} & 0 & 0 \\
0 & 0 & 0 & \xi^{3} & 0 \\
0 & 0 & 0 & 0 & \xi^{4}
\end{array}\right)
$$

3.3 $\left(\sigma_{3}\right)$ : Finally, let us consider the automorphism $\sigma_{3}$. Using the same arguments as in 3.2, elements $c_{i} \in S L\left(V_{i}\right)(i=1,2)$ can be found, with $c_{i}^{5}=1$ and no repeated eigenvalues, such that

$$
\left.\sigma_{3}\right|_{\mathfrak{s} l\left(V_{i}\right)}=\operatorname{Ad}_{c_{i}},\left.\quad \sigma_{3}\right|_{\mathfrak{g}_{\overline{1}}}=c_{1} \otimes \wedge^{2} c_{2}
$$

As $\sigma_{2}$ and $\sigma_{3}$ commute, it follows in particular that $\operatorname{Ad}_{b_{i}} \operatorname{Ad}_{c_{i}}=\operatorname{Ad}_{c_{i}} \operatorname{Ad}_{b_{i}}$ in $\mathfrak{s l}\left(V_{i}\right)$ or that $b_{i} c_{i}=\mu_{i} c_{i} b_{i}$ for some $0 \neq \mu_{i} \in \mathbb{F}$. Since $b_{i}^{5}=1$, we have $\mu_{i}^{5}=1, i=1,2$.

But if $\mu_{i}$ were equal to 1 , then $c_{i}$ would belong to $\left\{x \in \mathfrak{g l}\left(V_{i}\right): x b_{i}=b_{i} x\right\}=$ $\operatorname{span}\left\{b_{i}^{j}: j=0, \ldots, 4\right\}$ and so the subspace $\left\{x \in \mathfrak{s l}\left(V_{i}\right): \sigma_{2}(x)=\sigma_{3}(x)=x\right\}=$ $\left\{x \in \mathfrak{s l}\left(V_{i}\right): x b_{i}=b_{i} x\right\}$ would have dimension 4 , while we have

$$
\left\{x \in \mathfrak{s l}\left(V_{i}\right): \sigma_{2}(x)=\sigma_{3}(x)=x\right\} \subseteq \operatorname{Fix}\left(\sigma_{1}, \sigma_{2}, \sigma_{3}\right)=\mathfrak{g}_{0}=0,
$$

a contradiction. Therefore, $\mu_{i} \neq 1, i=1,2$.

We may change $\sigma_{3}$ to $\sigma_{3}^{j}$ for $1 \leq j \leq 4$, which implies changing $c_{i}$ to the corresponding power, and in this way we may assume that $\mu_{1}=\xi$, the fixed primitive fifth root of unity we have been using so far. (Note that the grading induced by $\sigma_{1}, \sigma_{2}, \sigma_{3}$ is induced also by $\sigma_{1}, \sigma_{2}$ and $\sigma_{3}^{j}$.)

Moreover, the commutativity of $\sigma_{2}$ and $\sigma_{3}$ on $\mathfrak{g}_{\overline{1}}$ gives

$$
b_{1} c_{1} \otimes \wedge^{2}\left(b_{2} c_{2}\right)=c_{1} b_{1} \otimes \wedge^{2}\left(c_{2} b_{2}\right)=\mu_{1} \mu_{2}^{2} b_{1} c_{1} \otimes \wedge^{2}\left(b_{2} c_{2}\right)
$$

so that $\mu_{1} \mu_{2}^{2}=1$, and thus we may assume that $\mu_{1}=\xi$ and $\mu_{2}=\xi^{2}$.

Since $b_{1} c_{1}=\xi c_{1} b_{1}$, we have $b_{1} c_{1}\left(v_{1 j}\right)=\xi^{j} c_{1}\left(v_{1 j}\right)(j=1, \ldots, 5)$, and hence we may scale the basic vectors $v_{1 j}$ so that $c_{1}\left(v_{1 j}\right)=v_{1(j+1)}$ for $j=1,2,3,4$. In other words, a basis can be taken in $V_{1}$ such that the coordinate matrices of $b_{1}$ and $c_{1}$ are

$$
b_{1} \leftrightarrow\left(\begin{array}{ccccc}
1 & 0 & 0 & 0 & 0 \\
0 & \xi & 0 & 0 & 0 \\
0 & 0 & \xi^{2} & 0 & 0 \\
0 & 0 & 0 & \xi^{3} & 0 \\
0 & 0 & 0 & 0 & \xi^{4}
\end{array}\right), \quad c_{1} \leftrightarrow\left(\begin{array}{ccccc}
0 & 0 & 0 & 0 & 1 \\
1 & 0 & 0 & 0 & 0 \\
0 & 1 & 0 & 0 & 0 \\
0 & 0 & 1 & 0 & 0 \\
0 & 0 & 0 & 1 & 0
\end{array}\right)
$$

In the same vein, since $b_{2} c_{2}=\xi^{2} c_{2} b_{2}$, by permuting and scaling the previous basic vectors on $V_{2}$ a new basis can be taken in $V_{2}$ such that the coordinate matrices of 
$b_{2}$ and $c_{2}$ are

$$
b_{2} \leftrightarrow\left(\begin{array}{ccccc}
1 & 0 & 0 & 0 & 0 \\
0 & \xi^{2} & 0 & 0 & 0 \\
0 & 0 & \xi^{4} & 0 & 0 \\
0 & 0 & 0 & \xi & 0 \\
0 & 0 & 0 & 0 & \xi^{3}
\end{array}\right), \quad c_{2} \leftrightarrow\left(\begin{array}{ccccc}
0 & 0 & 0 & 0 & 1 \\
1 & 0 & 0 & 0 & 0 \\
0 & 1 & 0 & 0 & 0 \\
0 & 0 & 1 & 0 & 0 \\
0 & 0 & 0 & 1 & 0
\end{array}\right) .
$$

In conclusion, up to equivalence, the only $\mathbb{Z}_{5}^{3}$-grading of $\mathfrak{g}$ such that $\operatorname{dim} \mathfrak{g}_{\alpha}=2$ for any $0 \neq \alpha \in \mathbb{Z}_{5}^{3}$ is given by the automorphisms $\sigma_{1}, \sigma_{2}, \sigma_{3}$ such that

$$
\begin{aligned}
& \sigma_{1}(x)=\xi^{i} x \quad \text { for any } x \in \mathfrak{g}_{\bar{\imath}} \text { and } 0 \leq i \leq 4, \\
& \left.\sigma_{2}\right|_{\mathfrak{g}_{\overline{1}}}=b_{1} \otimes \wedge^{2} b_{2}, \\
& \left.\sigma_{3}\right|_{\mathfrak{g}_{\overline{1}}}=c_{1} \otimes \wedge^{2} c_{2},
\end{aligned}
$$

where the $\mathfrak{g}_{\imath}$ 's are the homogeneous components in (2.3) and, on fixed bases of $V_{1}$ and $V_{2}, b_{1}$ and $c_{1}$ (respectively $b_{2}$ and $c_{2}$ ) are the endomorphisms of $V_{1}$ (respectively $\left.V_{2}\right)$ in (2.6) (respectively (2.7)).

Remark 2.9. The proof of the previous theorem gives a precise model for the $\mathbb{Z}_{5}^{3}$ Jordan grading of $E_{8}$.

\section{3. $\mathbb{Z}_{3}^{3}$-GRADINGS ON $E_{6}$ AND $F_{4}$}

In this section parts (ii) and (vi) of the Main Theorem will be proved. Many arguments are quite similar to the ones used for $E_{8}$, so they will just be sketched.

We start with $E_{6}$ :

Theorem 3.1. Let $\mathbb{F}$ be an algebraically closed field of characteristic 0 and let $\mathfrak{g}$ be the simple Lie algebra of type $E_{6}$ over $\mathbb{F}$. Then up to equivalence there is a unique $\mathbb{Z}_{3}^{3}$-grading of $\mathfrak{g}$ such that $\operatorname{dim} \mathfrak{g}_{\alpha}=3$ for any $0 \neq \alpha \in \mathbb{Z}_{3}^{3}$.

Proof. The same steps as for $E_{8}$ will be followed.

Step 1. The construction of a suitable model of the simple Lie algebra of type $E_{6}$.

Here the model appears in [Ada96, Chapter 13] (see also [Dra08, §3]). Let $V_{1}$, $V_{2}$ and $V_{3}$ be three vector spaces of dimension 3 over $\mathbb{F}$ and consider the $\mathbb{Z}_{3}$-graded Lie algebra

$$
\mathfrak{g}=\mathfrak{g}_{\overline{0}} \oplus \mathfrak{g}_{\overline{1}} \oplus \mathfrak{g}_{\overline{2}},
$$

where

$$
\begin{aligned}
& \mathfrak{g}_{\overline{0}}=\mathfrak{s l}\left(V_{1}\right) \oplus \mathfrak{s l}\left(V_{2}\right) \oplus \mathfrak{s l}\left(V_{3}\right), \\
& \mathfrak{g}_{\overline{1}}=V_{1} \otimes V_{2} \otimes V_{3}, \\
& \mathfrak{g}_{\overline{2}}=V_{1}^{*} \otimes V_{2}^{*} \otimes V_{3}^{*} .
\end{aligned}
$$

This is a $\mathbb{Z}_{3}$-graded Lie algebra, with the natural action of the semisimple algebra $\mathfrak{g}_{\overline{0}}$ on each of the other homogeneous components and where the brackets between elements in different components are given by suitable scalar multiples of the only $\mathfrak{g}_{0}$-invariant possibilities. In this way, $\mathfrak{g}$ is the exceptional simple Lie algebra of type $E_{6}$.

Step 2. Up to conjugation in Aut $\mathfrak{g}$, there is a unique order 3 automorphism of the simple Lie algebra $\mathfrak{g}$ of type $E_{6}$ such that the dimension of the subalgebra of fixed elements is 24 . 
Actually, this automorphism $\sigma$ is the one that corresponds to the only node labeled by 3 in the affine Dynkin diagram $E_{6}^{(1)}$ :

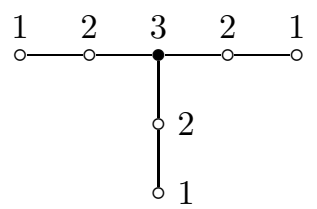

The uniqueness shows us that, up to conjugation, $\sigma$ is the automorphism of $\mathfrak{g}$ such that its restriction to $\mathfrak{g}_{\bar{\imath}}$ (with notation as in Step 1) is $\omega^{i}$ times the identity, where $\omega$ is a fixed primitive third root of unity.

Step 3. Assume that $\mathfrak{g}=\bigoplus_{0 \neq \alpha \in \mathbb{Z}_{3}^{3}} \mathfrak{g}_{\alpha}$ is a $\mathbb{Z}_{3}^{3}$-graded simple Lie algebra of type $E_{6}$ with $\operatorname{dim} \mathfrak{g}_{\alpha}=3$ for any $0 \neq \alpha \in \mathbb{Z}_{3}^{3}$. The homogeneous spaces are given by the common eigenspaces of three commuting order 3 automorphisms $\sigma_{1}, \sigma_{2}$, and $\sigma_{3}$ of $\mathfrak{g}$ which generate a subgroup of Aut $\mathfrak{g}$ isomorphic to $\mathbb{Z}_{3}^{3}$.

3.1 $\left(\sigma_{1}\right)$ : Step 2 shows us that, without loss of generality, we may assume that $\sigma_{1}$ is the automorphism such that $\sigma_{1}(x)=\omega^{i} x$ for any $x \in \mathfrak{g}_{\bar{\imath}}$ (notation as in Step 1). $3.2\left(\sigma_{2}\right)$ : As for $E_{8}$, the restriction $\left.\sigma_{2}\right|_{\mathfrak{g}_{0}}$ is an order 3 automorphism (otherwise the dimension of $\operatorname{Fix}\left(\sigma_{1}, \sigma_{2}\right)$ would be $\left.>6\right)$. Now, $\sigma_{2}$ induces a permutation of the three simple ideals of $\mathfrak{g}_{\overline{0}}$ of order 1 or 3 , but if the order were 3 , then the eight dimensional subspace $\left\{x+\sigma_{2}(x)+\sigma_{2}^{2}(x): x \in \mathfrak{s l}\left(V_{1}\right)\right\}$ would be contained in the six dimensional subspace $\operatorname{Fix}\left(\sigma_{1}, \sigma_{2}\right)$, a contradiction. Therefore, $\sigma_{2}$ leaves invariant $\mathfrak{s l}\left(V_{i}\right)$ for all $i$.

Now the same arguments as for $E_{8}$ show that one may find elements $b_{i} \in S L\left(V_{i}\right)$, $i=1,2,3$, such that $b_{i}^{3}=1$ and

$$
\left.\sigma_{2}\right|_{\mathfrak{s} l\left(V_{i}\right)}=\operatorname{Ad}_{b_{i}} \quad(i=1,2,3),\left.\quad \sigma_{2}\right|_{\mathfrak{g}_{\overline{1}}}=b_{1} \otimes b_{2} \otimes b_{3} .
$$

Moreover, the minimal polynomial of $b_{i} \in S L\left(V_{i}\right)$ is exactly $X^{3}-1$ (its eigenvalues have multiplicity 1$)$.

$3.3\left(\sigma_{3}\right):$ In the same vein, there are endomorphisms $c_{i} \in S L\left(V_{i}\right), i=1,2,3$, with minimal polynomial $X^{3}-1$ such that

$$
\left.\sigma_{3}\right|_{\mathfrak{g} \mathfrak{s} l\left(V_{i}\right)}=\operatorname{Ad}_{c_{i}},\left.\quad \sigma_{3}\right|_{\mathfrak{g}_{\overline{1}}}=c_{1} \otimes c_{2} \otimes c_{3} .
$$

As for $E_{8}$, the commutation of $\sigma_{2}$ and $\sigma_{3}$ and a dimension count show that $b_{i} c_{i}=$ $\mu_{i} c_{i} b_{i}$, with $1 \neq \mu_{i} \in \mathbb{F}$ and $\mu_{i}^{3}=1(i=1,2,3)$. Hence $\mu_{i} \in\left\{\omega, \omega^{2}\right\}$. Replacing $\sigma_{3}$ by $\sigma_{3}^{2}$ if necessary, it can be assumed that $\mu_{1}=\omega$.

Moreover, the commutativity of $\sigma_{2}$ and $\sigma_{3}$ on $\mathfrak{g}_{\overline{1}}$ forces the equality $\mu_{1} \mu_{2} \mu_{3}=1$ or $\mu_{2} \mu_{3}=\omega^{2}$. We conclude that $\mu_{1}=\mu_{2}=\mu_{3}=\omega$. Hence, a basis can be chosen on each $V_{i}$ such that the coordinate matrices of $b_{i}$ and $c_{i}$ are

$$
b_{i} \leftrightarrow\left(\begin{array}{ccc}
1 & 0 & 0 \\
0 & \omega & 0 \\
0 & 0 & \omega^{2}
\end{array}\right), \quad c_{i} \leftrightarrow\left(\begin{array}{ccc}
0 & 0 & 1 \\
1 & 0 & 0 \\
0 & 1 & 0
\end{array}\right)
$$


In conclusion, up to equivalence, the only $\mathbb{Z}_{3}^{3}$-grading of $\mathfrak{g}$ such that $\operatorname{dim} \mathfrak{g}_{\alpha}=3$ for any $0 \neq \alpha \in \mathbb{Z}_{3}^{3}$ is given by the automorphisms $\sigma_{1}, \sigma_{2}, \sigma_{3}$ such that

$$
\begin{aligned}
& \sigma_{1}(x)=\omega^{i} x \quad \text { for any } x \in \mathfrak{g}_{\bar{\imath}} \text { and } i=0,1,2, \\
& \left.\sigma_{2}\right|_{\mathfrak{g}_{\overline{1}}}=b_{1} \otimes b_{2} \otimes b_{3}, \\
& \left.\sigma_{3}\right|_{\mathfrak{g}_{\overline{1}}}=c_{1} \otimes c_{2} \otimes c_{3},
\end{aligned}
$$

where the $\mathfrak{g}_{\bar{\imath}}$ 's are the homogeneous components in (3.3) and, on fixed bases of $V_{1}$, $V_{2}$ and $V_{3}, b_{i}$ and $c_{i}$ are the endomorphisms of $V_{i}$ in (3.4).

The corresponding result for $F_{4}$ is the following:

Theorem 3.5. Let $\mathbb{F}$ be an algebraically closed field of characteristic 0 and let $\mathfrak{g}$ be the simple Lie algebra of type $F_{4}$ over $\mathbb{F}$. Then up to equivalence there is a unique $\mathbb{Z}_{3}^{3}$-grading of $\mathfrak{g}$ such that $\operatorname{dim} \mathfrak{g}_{\alpha}=2$ for any $0 \neq \alpha \in \mathbb{Z}_{3}^{3}$.

Proof. Here we will be even more sketchy, since the situation is simpler.

Just consider the model of $E_{6}$ obtained above, and consider the order 2 automorphism $\tau$ which permutes $V_{2}$ and $V_{3}$. The subalgebra $\mathfrak{g}$ of elements fixed by $\tau$ is a simple Lie algebra of type $F_{4}$ (see [Dra08, §3]). Therefore, $\mathfrak{g}$ appears as the $\mathbb{Z}_{3}$-graded Lie algebra

$$
\begin{aligned}
\mathfrak{g} & =\mathfrak{g}_{\overline{0}} \oplus \mathfrak{g}_{\overline{1}} \oplus \mathfrak{g}_{\overline{2}}, \\
\mathfrak{g}_{\overline{0}} & =\mathfrak{s l}\left(V_{1}\right) \oplus \mathfrak{s l}\left(V_{2}\right), \\
\mathfrak{g}_{\overline{1}} & =V_{1} \otimes S^{2}\left(V_{2}\right), \\
\mathfrak{g}_{\overline{2}} & =V_{1}^{*} \otimes S^{2}\left(V_{2}^{*}\right) .
\end{aligned}
$$

Here $S^{2}(V)$ denotes the subspace of symmetric tensors in $V \otimes V$.

Up to conjugation in Aut $\mathfrak{g}$, there is a unique order 3 automorphism of the simple Lie algebra $\mathfrak{g}$ of type $F_{4}$ such that the dimension of the subalgebra of fixed elements is 16 . Actually, this automorphism $\sigma$ is the one that corresponds to the only node labeled by 3 in the affine Dynkin diagram $F_{4}^{(1)}$ :

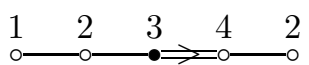

Now, the same types of arguments as for $E_{6}$ give the result.

Again, the proofs of Theorems 3.1 and 3.5 give precise models of the corresponding Jordan gradings. In [DM07, $\S 7]$ it was shown that for $F_{4}$ this grading is fine. These models are different from those obtained in [Eld08, §5.3], which were based on a $\mathbb{Z}_{3}^{2}$-grading of the Okubo algebra over $\mathbb{F}$, complemented by an extra order three automorphism induced by the triality automorphism associated to the Okubo algebra. For $E_{6}$, this unique $\mathbb{Z}_{3}^{3}$-grading is not fine, as the construction of $E_{6}$ in terms of an Okubo algebra requires the use of another two dimensional symmetric composition algebra, which in turn can be graded over $\mathbb{Z}_{3}$ and used to get a $\mathbb{Z}_{3}^{4}$-grading on $E_{6}$ (see [Eld08, for details). 


\section{REFERENCES}

[Ada96] J. F. Adams, Lectures on exceptional Lie groups, Chicago Lectures in Mathematics, University of Chicago Press, Chicago, 1996. MR1428422 (98b:22001)

[Ale74] A.V. Alekseevskiŭ, Finite commutative Jordan subgroups of complex simple Lie groups, Funkcional. Anal. i Priložen. 8 (1974), no. 4, 1-4. (English translation: Functional Anal. Appl. 8 (1974), no. 4, 277-279.) MR0379748 (52:653)

[Dra05] C. Draper, Models of $E_{8}$, communication presented at the International Mediterranean Congress of Mathematics, Almería (Spain), 2005.

[Dra08] _ Models of the Lie algebra $F_{4}$, Linear Algebra Appl. 428 (2008), no. 11-12, 2813-2839. MR2416591 (2009e:17015)

[DM07] C. Draper and C. Martín, Gradings on the Albert algebra and on $\mathfrak{f}_{4}$, Rev. Mat. Iberoamericana 25 (2009), no. 3, 841-908.

[Eld98] A. Elduque, Gradings on octonions, J. Algebra 207 (1998), no. 1, 342-354. MR.1643126 (99g:17055)

[Eld08] Gradings on symmetric composition algebras, to appear in J. Algebra, arXiv:0809.1922 [math.RA].

[Hes82] W.H. Hesselink, Special and pure gradings of Lie algebras, Math. Z. 179 (1982), no. 1, 135-149. MR643052 (83c:17025)

[Jac62] N. Jacobson, Lie algebras, Interscience Publishers, New York-London, 1962. MR0143793 (26:1345)

[Kac90] V.G. Kac, Infinite dimensional Lie algebras, 3rd edition, Cambridge University Press, Cambridge, 1990. MR 1104219 (92k:17038)

[KMRT98] M.-A. Knus, A. Merkurjev, M. Rost, and J.-P. Tignol, The book of involutions, American Mathematical Society Colloquium Publications, vol. 44, American Mathematical Society, Providence, RI, 1998. MR1632779 (2000a:16031)

[Kos08] B. Kostant, On some of the mathematics in Garrett Lisi's E(8) Theory of Everything, communication presented at the University of California Lie Theory Workshop, San Diego, CA, February 2008.

[KKU81] A.I. Kostrikin, I.A. Kostrikin, and V.A. Ufnarovskii, Orthogonal decompositions of simple Lie algebras, Soviet Math. Dokl. 24 (1981), no. 2, 292-296. MR0631924 (83c:17013)

[KP94] A.I. Kostrikin and P.H. Tiệp, Orthogonal decompositions and integral lattices, de Gruyter Expositions in Mathematics, vol. 15, Walter de Gruyter \& Co., Berlin, 1994. MR:1308713 (96f:17001)

[Oku78] S. Okubo, Pseudo-quaternion and pseudo-octonion algebras, Hadronic J. 1 (1978), no. 4, 1250-1278. MR510100 (80a:17004)

[OV91] A.L. Onishchik and E.B. Vinberg, Lie groups and Lie algebras. III, Encyclopaedia of Mathematical Sciences, vol. 41, Springer-Verlag, Berlin, 1994. A translation of Current problems in mathematics. Fundamental directions. Vol. 41 (Russian), Akad. Nauk SSSR, Vsesoyuz. Inst. Nauchn. i Tekhn. Inform., Moscow, 1990. MR.1349140 (96d:22001)

[Tho76] J.G. Thompson, A conjugacy theorem for $E_{8}$, J. Algebra 38 (1976), no. 2, 525-530. MR0399193(53:3044)

Departamento de Matemáticas e Instituto Universitario de Matemáticas y AplicaCiones, Universidad de Zaragoza, 50009 Zaragoza, Spain

E-mail address: elduque@unizar.es 